\section{Effects of a New Wound Dressing Material SG-01 in an Experimental Rat Skin Burn and Decubitus Ulcer Model}

\author{
Tetsuo Kaneko, ${ }^{*}, a, b$ Atsushi Hashimoto, ${ }^{a}$ \\ Norimitsu Umehara, ${ }^{a}$ and Masakatsu Tezuka ${ }^{b}$
}

${ }^{a} S S P$ Co., Ltd. Life Science Institute, 1143 Nanpeidai, Narita, Chiba 286-8511, Japan and ' Department of Health Science, College of Pharmacy, Nihon University, 7-7-1 Narashinodai, Funabashi, Chiba 274-8555, Japan

(Received April 1, 2006; Accepted May 8, 2006; Published online May 30, 2006)

A wound dressing material, SG-01 was developed to treat various skin ulcers. In this study, to clarify the features of SG-01, we investigated the therapeutic effects of this dressing material in rat burn and decubitus ulcer models. In the rat burn model, SG-01 significantly reduced the percent wound area compared to gauze (control group), and decreased the sum of the percent area, suggesting that SG-01 promotes the healing of burns. Subsequently, we investigated the curative effects of SG-01 in the rat decubitus ulcer model using commercially available dressing materials, $\mathrm{NU}$ GEL $^{\oplus, 2)}$ and DuoACTIVE CGF ${ }^{\circledR, 3(4)}$ SG-01 significantly reduced the percent wound area compared to gauze (control group), decreased the sum of the percent area, and shortened the interval until healing. There were no significant differences between SG-01 and the above commercially available materials. These results suggest that SG-01 is useful for treating burn and decubitus ulcers.

Key words — wound dressing, hydrogel, rat, burn model, decubitus ulcer model

\section{INTRODUCTION}

SG-01 is a wound dressing material consisted by monolithic adhesive including water and ionic cross-linking hydrophilic polymers, cellulose derivative and polyacrylate, with aluminum ion, spread onto the backing material.

\footnotetext{
*To whom correspondence should be addressed: SSP Co., Ltd. Life Science Institute, 1143 Nanpeidai, Narita, Chiba 286-8511, Japan. Tel.: +81-476-27-1511; Fax: +81-476-26-7948; E-mail: tetsuo.kaneko@ssp.co.jp
}

As this agent has favorable physical properties such as high water absorption, water-maintaining ability, appropriate vapor permeability, adhesiveness, oxygen permeability and flexibility, it is useful for removing exudate, and was developed to treat burns and decubitus ulcer. ${ }^{5)}$ Furthermore, SG-01 did not cause reactions in a primary skin irritation test, a cumulative skin irritation test, an intracutaneous reactivity test, a skin sensitization test, and a pyrogen test, suggesting its safety. ${ }^{6}$

In this study, we examined the pharmacological effects of SG-01 in rat burn and decubitus ulcer models.

\section{MATERIALS AND METHODS}

Test Substance — SG-01 $(2.5 \times 2.5 \mathrm{~cm})$ was used as a test substance, and gauze $(2.5 \times 2.5 \mathrm{~cm})$ containing sterile physiological saline was used as a control substance. In an experimental rat decubitus ulcer model, commercially available materials, NUGEL $^{\circledR}$ (hydrogel A; JOHNSON \& JOHNSON MEDICAL Inc., Tokyo, Japan) and DuoACTIVE CGF $^{\circledR}$ (hydrocolloid B; Bristle Myers Squibb Co., Ltd., Tokyo, Japan) $(2.5 \times 2.5 \mathrm{~cm})$, were also employed as commercially available control substance for comparison.

Animals — The experimental protocol was approved by the Ethics Review Committee for Animal Experimentation of Nihon University. 7-weekold male $\mathrm{Crj}$ : $\mathrm{CD}(\mathrm{SD}) \mathrm{IGS}(\mathrm{SPF})$ rats were obtained from Japan Charles River Co., Ltd. (Kanagawa, Japan). Pelleted chow (CRF-1, Oriental Yeast Industry Co., Ltd., Tokyo, Japan) and tap water were given ad libitum. These animals were acclimated for 1 week under the following conditions: temperature, $22 \pm 3^{\circ} \mathrm{C}$; humidity, $50 \pm 20 \%$; lighting cycle, $12 \mathrm{hr}$ / day; and ventilation cycle, 13 to 17 times/hr. The animals used were without abnormalities in the general condition.

\section{Experimental Methods}

Preparation of Models:

Burn Model: In 8-week-old rats, hair on the back was removed using electric hair clippers and an electric shaver. Under ether anesthesia, a burning iron weighing 52 to $54 \mathrm{~g}$ (a modified $100 \mathrm{~W}$ soldering iron; 302 to $303^{\circ} \mathrm{C}$; diameter, $10 \mathrm{~mm}$; contact pressure, 63.7 to $76.4 \mathrm{~g} / \mathrm{cm}^{2}$ ) was applied on an area on the posterior side $2-\mathrm{cm}$ distant from the right axilla and on the right side $1.5-\mathrm{cm}$ distant from the median line on the back, for $10 \mathrm{sec}$ to induce grade 
III burns (heat degeneration involving subcutaneous tissue). ${ }^{7)}$ After 2 days, necrotic tissue (skin) was resected under ether anesthesia, and used as a burn model. ${ }^{8,9)}$

Decubitus Ulcer Model: In 8-week-old rats, hair on the skin of the right greater trochanter was extensively removed using electric hair clippers and an electric shaver. For anesthesia, sodium pentobarbital at $50 \mathrm{mg} / \mathrm{kg}$ and sodium amobarbital at $30 \mathrm{mg} / \mathrm{kg}$ were intraperitoneally administered. On a wooden fixation plate, the animals were fixed in a prone position. As a cushion, a piece of absorbent cotton was placed between the femoral region and the fixation plate, and a stainless steel stick weighing 1.02 to $1.03 \mathrm{~kg}$ and measuring $19 \mathrm{~mm}$ and $50 \mathrm{~cm}$ in diameter and length, respectively, with a gum stopper measuring $12 \mathrm{~mm}$ in diameter, was placed on the skin of the right greater trochanter for 24-hr pressure loading ( 902.3 to $911.2 \mathrm{~g} / \mathrm{cm}^{2}$ ). If necessary, additional anesthesia with ether, sodium pentobarbital, or sodium amobarbital was performed during pressure loading. After $24 \mathrm{hr}$, pressure loading was removed, and if necessary, $5 \%$ glucose was orally administered. After 2 days, the necrotic skin was resected under ether anesthesia in animals with a good general condition and a round necrotic site, and used as a decubitus ulcer (pressure-loaded necrosis) model. ${ }^{8,9)}$

Grouping: Resected specimens (wound surface) from the burn and decubitus ulcer models were washed with absorbent cotton containing sterile physiological saline. The maximum and minimum diameters of the wound surface were measured using calipers to calculate the wound area (maximum diameter $\times$ minimum diameter). In the burn model, 16 animals were selected, and divided into 2 groups so that the wound area was similar between the two groups. In the decubitus ulcer model, 40 animals were selected, and divided into 4 groups so that the wound area was similar between the four groups.

Application of the Test Substance: After grouping, the test substance was applied on the wound surface, and fixed with an elastic bandage (Elastopore $^{\circledR}$, Nichiban Co., Ltd., Tokyo, Japan). In the control group, 2 sheets of gauze $(2.5 \times 2.5 \mathrm{~cm})$ containing sterile physiological saline were applied, and similarly fixed.

The test and control substance were changed once a day. Before application, the wound surface was wiped with absorbent cotton containing sterile physiological saline. These procedures were performed under ether anesthesia until the macroscopic completion of epithelization of the wound surface.

Observation and Evaluation Methods: After the test substance was applied, the maximum and minimum diameters of the wound surface were measured using calipers once a day to calculate the wound area. Measurement was continued until epithelization was completed. Furthermore, we calculated the percent wound area (\%) to the area at grouping (when the burn and decubitus ulcer models were prepared), and the sum of the percent area (area under the percent wound area curve) using the following formula. The interval from application of the test substance until the completion of epithelization was regarded as the interval until healing:

Percent wound area $(\%)=[($ maximum diameter $\times$ minimum diameter on the day of measurement)/ (maximum diameter $\times$ minimum diameter at the start of application) $] \times 100$

Sum of the percent area $(\% \times$ day $)=\Sigma[($ percent wound area after $n$ days + percent wound area after $(n+1)$ days $) / 2]$.

$n$ : Interval from the start of application until the day before healing.

Measurement of Body Weight: During the study period, body weight was measured at 3- to 4-day intervals.

Statistical Analysis: The data are expressed as the mean \pm standard deviation. Significance was tested using the F-test. When isovariance was noted, Student's $t$-test was employed. When unequal variance was noted, Aspin-Welch's $t$-test was used. $p<0.05$ was regarded as significant.

In the decubitus ulcer model, the multiple comparison test was performed with consider to the percent wound area, sum of the percent area, and interval until healing, as described below, to compare the efficacy between SG-01 and the two commercially available dressing materials. Isovariance was tested using Bartlett's method $(p<0.05)$. When isovariance was noted, one-way analysis was performed. When there was a significant difference, the means were compared using Tukey's method. When unequal variance was noted, the Kruskal-Wallis H-test was employed. When there was a significant difference, the means were compared using Tukey's method. $p$ $<0.05$ was regarded as significant.

Effect (a decrease in the sum of the percent area and shortening of the interval until healing) on the decubitus ulcer model was compared based on $90 \%$ confidence intervals for differences in the mean logarithm [criteria: $\log (0.8)$ to $\log (1.25)$ ], as described for the test methods for biological equivalence. ${ }^{10,11)}$ 


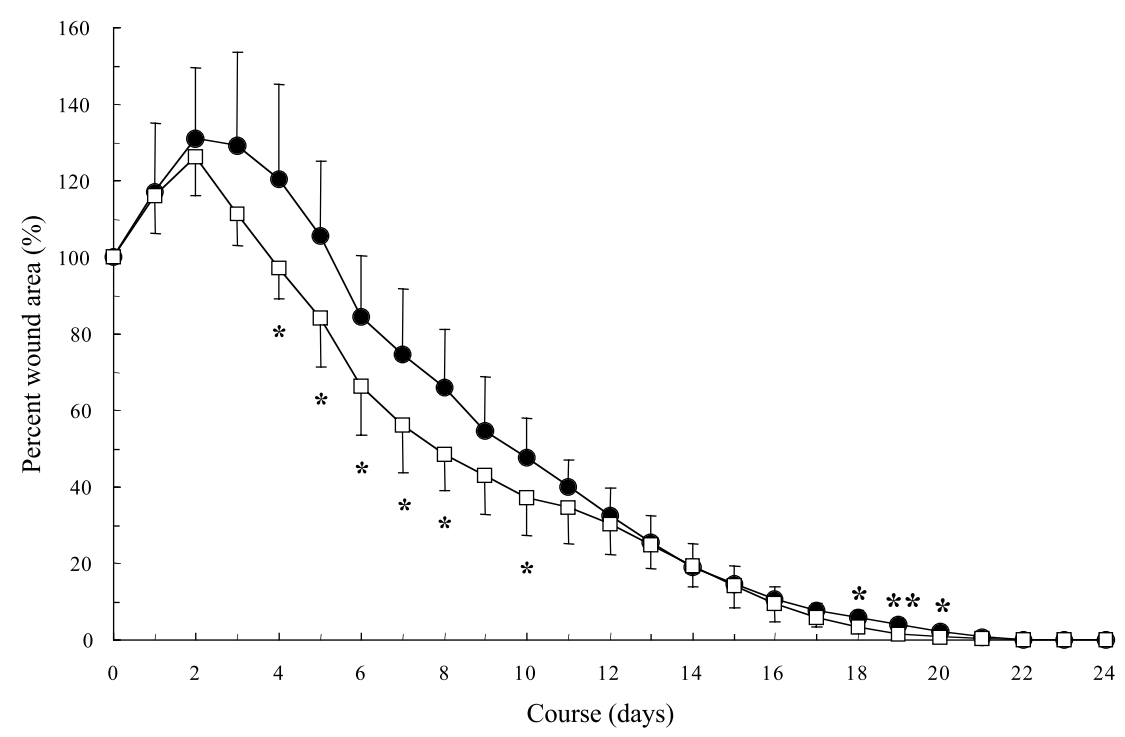

Fig. 1. Effect of SG-01 in the Burn Model - Percent Wound Area -—: Control, — $\square$ —: SG-01, *, **: $p<0.05,0.01$ vs. Control.

Table 1. Effect of SG-01 in Rat Burn Model — Sum of the Percent Area, Interval Until Healing -

\begin{tabular}{llcc}
\hline \hline & $n$ & Sum of the percent area $(\% \times$ days $)$ & Interval until healing (days) \\
\hline Control & 8 & $1142.3 \pm 176.7$ & $22.5 \pm 0.9$ \\
SG-01 & 8 & $979.0 \pm 114.6^{*}$ & $22.0 \pm 1.1$ \\
\hline
\end{tabular}

Each value represents mean \pm S.D. Significant difference from control $\left({ }^{*} p<0.05\right)$.

\section{RESULTS}

\section{Effects on Experimental Burns}

Figure 1 shows serial changes in the percent wound area.

In the control group, the percent wound area gradually increased to $131.0 \pm 18.4 \%$ after 2 days of application, but then decreased. In the SG-01 group, the percent wound area similarly increased for 2 days of application, but then decreased. The percent wound area in this group was lower than that in the control group; it was significantly lower on days 4 to 8,10 , and 18 to 20 of application.

Table 1 shows the sum of the percent area (area under the percent wound area curve) and the interval until healing. In the control group, the sum of the percent area was $1142.3 \pm 176.7 \% \times$ days. In the SG-01 group, the value was $979.0 \pm 114.6 \% \times$ days, significantly lower than the control value.

The interval until healing was $22.5 \pm 0.9$ and $22.0 \pm 1.1$ days in the control and SG-01 groups, respectively.

Body weight did not change until day 6 in the control or SG-01 groups, and gradually increased after day 9 (data not shown).

\section{Effects on Experimental Decubitus Ulcers}

Figure 2 shows serial changes in the percent wound area. In the control group, the percent wound area gradually decreased after one day of application. In the SG-01 group, the percent wound area also gradually decreased after one day of application. It was significantly lower than the control value from day 3 until 21. In the hydrogel A and hydrocolloid B groups, similar to SG-01; the percent wound area was significantly lower than the control value from day 3 until 21.

Table 2 shows the sum of the percent area (area under the percent wound area curve) and the interval until healing.

In the control group, the sum of the percent area was $901.8 \pm 42.6 \% \times$ days. In the SG-01 group, the value was $727.0 \pm 76.6 \% \times$ days, significantly lower than the control value. In the hydrogel A and hydrocolloid B groups, the values were $722.2 \pm 56.6$ and $710 \pm 60.1 \% \times$ days, respectively, significantly lower than the control value. The interval until healing was $22.2 \pm 0.9$ and $20.8 \pm 1.2$ days in the control and 


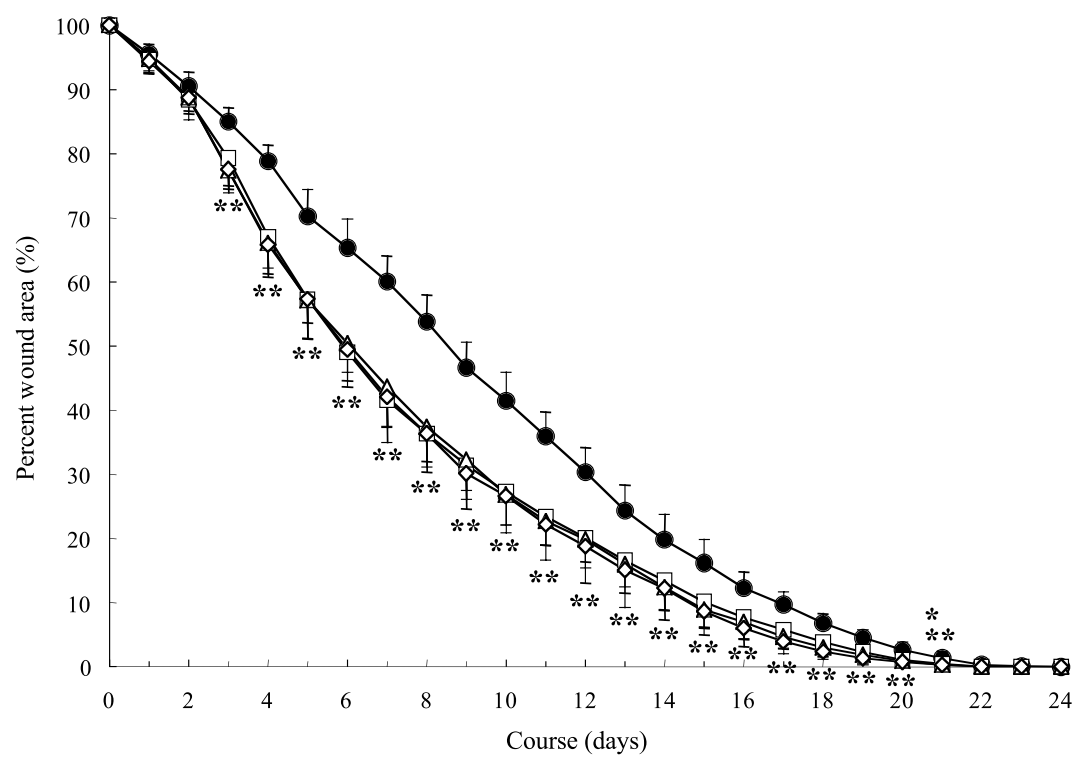

Fig. 2. Effect of SG-01 in the Decubitus Ulcer Model - Percent Wound Area -

$\multimap$ —: Control, $\square \square$ : SG-01, $\backsim$ : hydrogel A, $\prec$ : hydrocolloid B. *, **: $p<0.05,0.01$ vs. Control.

Table 2. Effect of SG-01 in Rat Decubitus Ulcer Model — Sum of the Percent Area, Interval Until Healing -

\begin{tabular}{llcc}
\hline \hline & $n$ & Sum of the percent area $(\% \times$ days $)$ & Interval until healing (days) \\
\hline Control & 10 & $901.8 \pm 42.6$ & $22.2 \pm 0.9$ \\
SG-01 & 10 & $727.0 \pm 76.6^{* *}$ & $20.8 \pm 1.2^{* *}$ \\
Hydrogel A & 10 & $722.2 \pm 56.6^{* *}$ & $21.0 \pm 1.1^{*}$ \\
Hydrocolloid B & 10 & $710.0 \pm 60.1^{* *}$ & $20.8 \pm 1.2^{* *}$ \\
\hline
\end{tabular}

Each value represents mean \pm S.D. Significant difference from control $(*, * * p<0.05,0.01)$.

Table 3. Analysis of Equivalence

\begin{tabular}{|c|c|c|c|c|}
\hline & \multicolumn{2}{|c|}{ SG-01 vs. hydrogel A } & \multicolumn{2}{|c|}{ SG-01 vs. hydrocolloid B } \\
\hline & $\begin{array}{l}\text { Sum of the } \\
\text { percent area }\end{array}$ & $\begin{array}{l}\text { Interval until } \\
\text { healing }\end{array}$ & $\begin{array}{l}\text { Sum of the } \\
\text { percent area }\end{array}$ & $\begin{array}{l}\text { Interval until } \\
\text { healing }\end{array}$ \\
\hline Parameter & logarithm & logarithm & logarithm & Logarithm \\
\hline Detection $1-\beta(\alpha=0.1, \nu=10)$ & $>0.99$ & $>0.99$ & $>0.99$ & $>0.99$ \\
\hline Minimum difference in detection & & & & \\
\hline$(\alpha=0.1,1-\beta=0.8)$ & $1.89 \%$ & $2.40 \%$ & $1.94 \%$ & $2.59 \%$ \\
\hline Difference in the mean & $-0.070 \%$ & $0.326 \%$ & $-0.336 \%$ & $0.000 \%$ \\
\hline 90\%onfidence interval & $0.926-1.070 \%$ & $0.968-1.054 \%$ & $0.908-1.054 \%$ & $0.955-1.047 \%$ \\
\hline Evaluation & equivalent & equivalent & equivalent & Equivalent \\
\hline
\end{tabular}

Analysis of equivalence were calculated from results in Table 2.

SG-01 groups, respectively, with a significant difference. In the hydrogel A and hydrocolloid B groups, the values were $21.0 \pm 1.1$ and $20.8 \pm$ 1.2 days, respectively, significantly lower than the control value.

Effect was compared among the SG-01, hydrogel A, and hydrocolloid B groups based on $90 \%$ confidence intervals for differences in the mean loga- rithm, as shown in Table 3. Effect was considered to be equivalent based on a decrease in the sum of the percent area and shortening of the interval until healing. In the control, SG-01, hydrogel A, and hydrocolloid B groups, body weight did not change until day 3 , and gradually increased after day 6 (data not shown). 


\section{DISCUSSION}

To clarify the pharmacological effects of SG01 , we investigated the effects of this material on burns and decubitus ulcers in experimental rat burn and decubitus ulcer models using the percent wound area, the sum of the percent area (area under the percent wound area curve), and the interval until healing as indices.

As it is important to maintain wet environments on the wound surface for wound healing, ${ }^{12-15}$ ) we employed rat burn and decubitus ulcer models, which reflect decubitus ulcers and burns in humans, and are commonly used in drug efficacy tests; rat burns and decubitus ulcers require a higher secretion of body fluid for the promotion of healing compared to cutting wounds, and the process of wound healing resembles that in humans. ${ }^{16,17)}$ Therefore, we considered that the study using burn and decubitus ulcer models was appropriate for evaluating the features of the wound dressing material.

In the burn model, the percent wound area after 4 to 8 days of application in the SG-01 group was significantly lower than that in the control group. The sum of the percent area in the SG-01 group was significantly lower than that in the control group. The interval until healing was shortened in the SG01 group. After 4 to 8 days of application, a relatively large volume of exudate containing various factors involved in wound healing, such as cell growth factor and various cytokines, is secreted. ${ }^{18)}$ Various covering agents consisting of hydrophilic colloid contribute to wound healing by accumulating exudate on the wound surface and promoting moisturization. ${ }^{19)}$ Therefore, SG-01 may have efficiently promoted retention of exudate in this phase, resulting in a significant decrease in the percent wound area. Thus, our results suggest that SG-01, which consists of a hydrophilic polymer, promotes wound healing by accumulating exudate containing various factors involved in wound healing, such as cell growth factor and various cytokines, on the wound surface.

Using the rat decubitus ulcer model, we compared the effects of SG-01 to those of hydrogel A and hydrocolloid $\mathrm{B}$. The percent wound area in the SG-01 group was significantly lower than that in the control group after 3 to 21 days of application. Similarly, the values in the hydrogel A and hydrocolloid B groups were significantly lower than that in the control group after 3 to 21 days of application. The sum of the percent area and the interval until heal- ing in the SG-01, hydrogel A, and hydrocolloid B groups were significantly lower than the control values. Thus, SG-01 promoted healing in the rat decubitus ulcer model. SG-01 decreased the percent wound area when a large volume of exudate was secreted ${ }^{18)}$ as demonstrated for hydrogel A and hydrocolloid $\mathrm{B}$, suggesting that the action mechanism of SG-01 in this model involves retention of exudate on the wound surface, ${ }^{19)}$ as demonstrated in the rat burn model.

These results suggest that SG-01 is useful for promoting wound healing in rat burn and decubitus ulcer models. Furthermore, SG-01 has favorable physical features ${ }^{5)}$ such as high water absorption, water-maintaining ability, appropriate vapor permeability, adhesiveness, oxygen permeability and flexiblity. In addition, after absorption of exudate, the adhesiveness of this agent in the wound site is reduced while maintaining the morphology of crosslinked gel, facilitating the exchange of the covering material and showing paste flexibility-related shock absorbing effects and cooling effects. Therefore, SG01 may improve compliance, and relieve pain in clinical practice.

\section{REFERENCES}

1) Kuwahara, H., Oura, T., Ikawa, H., Kokubu, I., Honda, K., Iida, K., Narumi, E., Ohkubo, Y. and Oiwa, A. (1993) Clinical study of synthetic wound dressing NG-01. Clin. Rep., 27, 4958-4968.

2) Karube, S., Sakamoto, H. and Seki, N. (1996) Clinical experience with $\mathrm{Nu}^{-\mathrm{Gel}^{\circledast}}{ }^{\circledR}$ hydrogel dressing in pressure sore. Clin. Rep., 30, 2311-2318.

3) Ishibashi, Y., Nakagawa, H., Iwata, M., Kurose, N., Azuma, Y., Saeki, H., Wakugawa, M., Watanabe, T., Kikuchi, K., Adachi, M., Harada, S., Nakanishi, H., Komine, M., Abe, M., Tomisawa, T., Nakauti, Y., Ohara, K. and Tsukada, M. (1993) Clinical study of DuoDERM $^{\circledR}$ CGF on skin ulcers. J. Clinical Therapeutics \& Medicines, 9, 443-454.

4) Yonemoto, K., Kondo, S., Asai, T., Ohkawa, T., Masuzawa, M., Kanamaru, T., Nishiyama, S., Takano, A. and Kawano, N. (1993) Clinical effect of DuoDERM ${ }^{\oplus}$ CGF dressing on skin ulcers. $J$. Clinical Therapeutics \& Medicines, 9, 455-463.

5) Kaneko, T., Otsuka, S., Narui, T., Umehara, N., Kasai, S. and Tezuka, M. (2004) Characteristics of novel hydrogel dressing material. Drug Delivery System, 19, 117-126.

6) Kaneko, T., Hashimoto, A., Hayashi, T., Umehara, N. and Tezuka, M. (2005) Safety studies of a new 
wound dressing material SG-01 for several experimental injury in rabbits and guinea pigs. Drug Delivery System, 20, 145-156.

7) Anazawa, S., Kuramoto, S., Tanase, S., Takao, Y. and Watanabe, S. (1995) Class and definition of wound. In Dressing, the new control method for wound, Herusu Shuppan Co., Inc., Tokyo, pp. 141148.

8) Kamidaki, H., Ohta, T., Murase, H., Ohta, Y., Ogawa, J., Aida, M., Ishida, M. and Kawashima, M. (1990) Effect of NI-009 on the healing of experimental dermal wounds. J. Clinical Therapeutics \& Medicines, 6, 627-638.

9) Yamashita, S., Ohmi, A., Hamada, Y., Oishi, K., Yano, M., Kuroki, S., Fuji, Y., Akamatsu, H. and Kobatake, H. (1989) Effect of ointment containing collagenase derived from Achromobacter iophagus (ACR-59 Ointment) upon burn, decubital ulcer, Open and cut wounds studied with experimental rat models. Pharmacometrics, 37, 313-327.

10) Aoyagi, N. (1997) Bioequivalence study in WHO and Japan. Iyakuhin Kenkyu, 28, 355-369.

11) Director, Evaluation and Licensing Devision, Pharmaceutical Bureau, Ministry of Health, Labour and Welfare. Guidelines for bioequivalence testing of generic drugs (Notification No. 487 of the Evaluation and Licensing Division, PMSB dated December 22, 1997, Notification No. 786 of the Evaluation and Licensing Division, PMSB dated May 31, 2001), Tokyo.
12) Winter, G. D. (1962) Formation of the scab and the rate of epithelization of superficial wounds in the skin of the young domestic pig. Nature (London), 193, 293-294.

13) Hinman, C. D. and Maibach, H. (1963) Effect of air exposure and occlusion on experimental human skin wounds. Nature (London), 200, 377-379.

14) Anazawa, S., Nagumo, Y. and sakurai, K. (1989) Wound healing. Current trend of wound management focusing on variety of dressing materials. Surgical Diagnosis \& Treatment, 31, 1-10.

15) Schultz, G., Sibbald, G., Falanga, V., Ayello, E., Dowesett, C., Harding, K., Romanelli, M., Stacey, M., Teoy, L. and Vanscheidt, W. (2003) Wound bed preparation : a systematic approach to wound managementet. Wound Repair Regen., 11, 1-28.

16) Kubochi, K. and Abe, O. (1992) Special issue: Clinical aspects and topics of wound healing. History and perspective of healing research. Significance for its clinical aspect. Surgical Diagnosis \& Treatment, 34, 1121-1124.

17) Imayama, S. (1997) Recent topics on wound healing. Basics of wound healing. Wound healing and arterialization. Kowa Medical Magazine, 1, 8-13.

18) Ono, I. and Kaneko, F. (1994) Evaluation of growth factors and cytokines in skin exudates. Fukushima Medical Journal, 44, 261-266.

19) Kimura, H. (1990) Our experience using TEGASORB $^{\mathrm{TM}}$ for pressure sores. Clin. Rep., 24, 6509-6514. 